\title{
ONE-THREE JOIN: A GRAPH OPERATION AND ITS CONSEQUENCES
}

\author{
M.A. Shalu and S. Devi Yamini \\ IIITD \& M, Kancheepuram, Chennai-600127, India \\ e-mail: shalu@iiitdm.ac.in \\ mat10d001@iiitdm.ac.in
}

\begin{abstract}
In this paper, we introduce a graph operation, namely one-three join. We show that the graph $G$ admits a one-three join if and only if either $G$ is one of the basic graphs (bipartite, complement of bipartite, split graph) or $G$ admits a constrained homogeneous set or a bipartite-join or a join. Next, we define $\mathcal{M}_{H}$ as the class of all graphs generated from the induced subgraphs of an odd hole-free graph $H$ that contains an odd anti-hole as an induced subgraph by using one-three join and co-join recursively and show that the maximum independent set problem, the maximum clique problem, the minimum coloring problem, and the minimum clique cover problem can be solved efficiently for $\mathcal{M}_{H}$.
\end{abstract}

Keywords: one-three join, bipartite-join, homogeneous set, odd hole-free graphs.

2010 Mathematics Subject Classification: 05C75, 05C76.

\section{REFERENCES}

[1] R.K. Ahuja, T.L. Magnanti and J.B. Orlin, Network Flows (Prentice-Hall, Englewood Cliffs, NJ, 1993) 409-411.

[2] M. Basavaraju, L.S. Chandran and T. Karthick, Maximum weight independent sets in hole- and dart-free graphs, Discrete Appl. Math. 160 (2012) 2364-2369. doi:10.1016/j.dam.2012.06.015

[3] D. Bienstock, On complexity of testing for odd holes and induced odd paths, Discrete Math. 90 (1991) 85-92. doi:10.1016/0012-365X(91)90098-M

[4] H.L. Bodlaender, A. Brandstädt, D. Kratsch, M. Rao and J. Spinrad, On algorithms for $\left\{P_{5}\right.$, gem $\}$-free graphs, Theoret. Comput. Sci. 349 (2005) 2-21. doi:10.1016/j.tcs.2005.09.026 
[5] A. Brandstädt and V. Giakoumakis, Addendum to: Maximum weighted independent sets in hole- and co-chair-free graphs, Inform. Process. Lett. 115 (2015) 345-350. doi:10.1016/j.ipl.2014.09.019

[6] A. Brandstädt, V. Giakoumakis and F. Maffray, Clique separator decomposition of hole- and diamond-free graphs and algorithmic consequences, Discrete Appl. Math. 160 (2012) 471-478.

doi:10.1016/j.dam.2011.10.031

[7] A. Brandstädt and T. Karthick, Weighted efficient domination in two subclasses of $P_{6}$-free graphs, Discrete Appl. Math. 201 (2016) 38-46. doi:10.1016/j.dam.2015.07.032

[8] A. Brandstädt and R. Mosca, Maximum weight independent sets in odd hole-free graphs without dart or without bull, Graphs Combin. 31 (2015) 1249-1262. doi:10.1007/s00373-014-1461-x

[9] M. Chudnovsky and P. Seymour, The structure of claw-free graphs, Surveys in Combinatorics, London Math. Soc. Lecture Note Ser. 327 (2005) 153-171.

[10] M. Chudnovsky, N. Robertson, P. Seymour and R. Thomas, The strong perfect graph theorem, Ann. of Math. 164 (2006) 51-229. doi:10.4007/annals.2006.164.51

[11] M. Chudnovsky, I. Penev, A. Scott and N. Trotignon, Substitution and $\chi$-boundedness, J. Combin. Theory Ser. B 103 (2013) 567-586. doi:10.1016/j.jctb.2013.02.004

[12] M. Conforti, G. Cornuéjols and K. Vušković, Even-hole-free graphs, Part II: Recognition algorithm, J. Graph Theory 40 (2002) 238-266. doi:10.1002/jgt.10045

[13] M. Conforti, G. Cornuéjols and K. Vušković, Decomposition of odd-hole-free graphs by double star cutsets and 2-joins, Discrete Appl. Math. 141 (2004) 41-91. doi:10.1016/S0166-218X(03)00364-0

[14] M. Conforti, G. Cornuéjols, X. Liu and K. Vušković and G. Zambelli, Odd-hole recognition in graphs of bounded clique size, SIAM J. Discrete Math. 20 (2006) $42-48$. doi:10.1137/S089548010444540X

[15] D.G. Corneil, H. Lerchs and L.S. Burlingham, Complement reducible graphs, Discrete Appl. Math. 3 (1981) 163-174. doi:10.1016/0166-218X(81)90013-5

[16] T. Feder, P. Hell, S. Klein and R. Motwani, Complexity of graph partition problems, in: Proceedings of the Thirty-First Annual ACM Symposium on Theory of Computing (1999) 464-472. doi:10.1145/301250.301373

[17] T. Feder, P. Hell, S. Klein and R. Motwani, List partitions, SIAM J. Discrete Math. 16 (2003) 449-478. doi:10.1137/S0895480100384055 
[18] S. Földes and P.L. Hammer, Split graphs, Congr. Numer. 19 (1977) 311-315.

[19] M. Grötschel, L. Lovász and A. Schrijver, Polynomial algorithms for perfect graphs, Ann. Discrete Math. 21 (1984) 325-356. doi:10.1016/s0304-0208(08)72943-8

[20] N.C. Lê, C. Brause and I. Schiermeyer, Extending the MAX Algorithm for maximum independent set, Discuss. Math. Graph Theory 35 (2015) 365-386. doi:10.7151/dmgt.1811

[21] R. Mosca, Stable sets for $\left(P_{6}, K_{2,3}\right)$-free graphs, Discuss. Math. Graph Theory 32 (2012) 387-401. doi:10.7151/dmgt.1598

[22] D.B. West, Introduction to Graph Theory (Prentice Hall, USA, 1996).

Received 29 September 2015

Revised 2 June 2016 Accepted 2 June 2016 